\title{
SOBRE TÉCNICA E IDEOLOGÍA
}

\author{
David Martín Solano \\ Doctorando, Universidad de La Rioja \\ es.david.martin@gmail.com
}

RESUMEN: La tecnología es una rama del saber que pertenece por completo al ámbito de la ciencia. Sin embargo, uno de sus fundamentos es ajeno a la cognición, a saber, la valoración de los fines. Ese elemento de la tecnología, allende la ciencia, es parte de la ideología, disciplina muy poco indagada. Es necesario ampliar el estudio de la racionalidad para engarzar ciencia e ideología, de manera que se pueda acometer cualquier cuestión axiológica.

Palabras clave: Bunge, técnica, ideología, ética, racionalidad.

\section{ON TECHNOLOGY AND IDEOLOGY}

ABSTRACT: Technology is a branch of knowledge which falls entirely within the scientific realm. However, it includes a noncognitive fundamental element, videlicet, the valuation of ends. This ethical part of technology is to be found beyond science, as a part of the largely uninquired discipline of ideology. A widening of the study of rationality is required to assemble both sciental and ideological subjets, in order to cope with any piece of axiological foundation.

Keywords: Bunge, technology, ideology, ethics, rationality.

Recibido: 6 de Noviembre de 2017 Aceptado: 14 de Diciembre de 2017

\section{Introducción}

Mario Bunge ha realizado una de las más completas y sólidas explicaciones de la tecnología de que disponemos. Sin embargo, no ha conseguido dar cuenta de un elemento fundamental de toda teoría técnica, a saber, la valoración hu- 
mana de los fines y los medios. Creemos que la solución a este y a otros problemas del conocimiento la proporciona un estudio de la racionalidad ampliado a sistemas mentales distintos de los puramente cognitivos y la consiguiente ampliación del saber a disciplinas intelectuales distintas de la ciencia y la técnica. Los valores humanos, ajenos a la axiología técnica, son elementos de la realidad y por tanto, en algunos respectos, competencia de la ciencia; pero también participan de la ideología y por tanto, en otros respectos, quedan más allá del dominio del conocimiento. Toda teoría técnica que no se ocupe de los valores humanos en tanto que elementos de la realidad y en tanto que componentes de una ideología quedará incompleta.

\section{La técnica}

La ciencia consiste en elaborar conocimiento acerca de todo lo que influye, directa o indirectamente, en la vida de las personas. La técnica consiste en diseñar y ejecutar procedimientos para manipular esos elementos del universo. El hombre «doma y modela la naturaleza para ajustarla a sus necesidades; construye la sociedad y es constuido por ella; intenta por tanto remodelar su entorno artificial para adpatarlo a sus necesidades y sueños animales y espirituales: crea el mundo de los artefactos y el mundo de la cultura» $(1959,28)^{1}$. Al igual que las ciencias, las técnicas son todas ellas fácticas (2006a, 270). Por eso, «no hay una línea nítida que divida los problemas científicos de los tecnológicos» (1967a, 208).

Resumimos -y retocamos- la tesis inicial de Ortega (1939). La técnica es el conjunto de los medios para hacer más efectiva, más eficiente o más eficaz la subvención de las necesidades y los caprichos. Lo que hace al ser humano realmente especial es que no solo intenta satisfacer sus necesidades, sino que también hace acopio de lo que contribuye a satisfacerlas; y no solo lo acopia, también lo produce. Debido a ello, la vida humana se ha llenado de actividades productivas en una proporción enorme.

Bunge extiende la relación entre ciencia y técnica hasta hacerla total: toda técnica se sirve de una o varias ciencias aplicadas; toda ciencia es también ciencia aplicada; toda ciencia aplicada es útil a una o varias técnicas. Aún no se ha alcanzado la combinatoria óptima porque hay varias ciencias a partir de las cuales aún no se han generado técnicas o ciencias aplicadas (2012a, 80). El conocimiento puede ser explícito o tácito; también puede ser proposicional (knowing what) o pericial (knowing how) (1983a, 77; 2012b, 72). Se puede conocer sin saber cómo hacer, y saber cómo hacer sin conocer. Bunge ofrece dos ejemplos: el ser humano se ha pasado milenios teniendo hijos sin saber la causa; hoy día se sabe mucho de las estrellas, pero no cómo hacerlas (2012b, 57).

1. Las citas en las que no se identifica al autor son de Mario Bunge. 
Hay un tipo de técnica que se denomina 'tecnología'. Se trata de una metatécnica, una "técnica de segundo orden", porque su cometido es elaborar herramientas o cualquier otro mecanismo a usar en una o varias técnicas. Algunos identifican la tecnología con la ingeniería, lo que es erróneo. Si fueran lo mismo, "no sabríamos dónde ubicar la bioingeniería, la tecnología educativa y otras disciplinas que no participan en la producción» (1976). Proponemos llamar 'ingeniero' tanto al tecnólogo que diseña el procedimiento de fabricar la herramienta como al menestral que ejectuta ese procedimiento.

Bunge restringe el concepto 'tecnología' al diseño que se fundamenta en el conocimiento científico: la tecnología es "la disciplina basada en la ciencia que se ocupa de diseñar (no de manufacturar) cosas y procesos artificiales» (1989b, 258). Por tanto, algunas herramientas y técnicas no son fruto de la tecnología, porque su elaboración consiste en un diseño rudimentario en el que intervienen nociones prácticas precientíficas (1985b, 42). Propone usar el término 'técnica' para estos mecanismos y el término 'tecnología' para los otros (1980a, 206; 1996, 280). Así, la tecnología es «el enfoque científico de los problemas prácticos, es decir, el manejo de esos problemas mediante el conocimiento científico y con la ayuda del método científico» $(1959,56)$. Objetamos a Bunge: aunque sean unos gnósicos y otros epistémicos, la tecnología la constituyen todos los diseños. Para denominar los mecanismos a los que Bunge asigna el término 'técnica' proponemos la palabra 'artesanía', que en ocasiones él también utiliza. Sí que tiene razón al propugnar que, igual que sucede con la ciencia, hay técnicas que evolucionarion de manera intuitiva, con un control sistemático pero no sistematizado de sus procesos. Hoy día, en cambio, toda técnica madura se fundamenta en la ciencia (1960a, 41).

La ciencia se llama 'aplicada' cuando se realiza para crear técnicas o para mejorar técnicas ya existentes. Cuando no es ciencia aplicada, se la llama 'bási$\mathrm{ca}^{\prime}$. Bunge distingue cuatro actividades: la ciencia básica, la ciencia aplicada, la técnica y la producción (1980a, 210; 1980b, 27; 1985b, 262, 264). Nuestra gestión conceptual y terminológica de las dos últimas ideas difiere de la bungeana. La tercera actividad la llamamos 'tecnología' y distinguimos la que consiste en la fabricación de artefactos y la que consiste en el diseño de procedimientos, estén asistidos o no por artefactos. Cabe apuntar que la fabricación de artefactos requiere a su vez del diseño de procedimientos para fabricarlos, y que no hay límite teórico, aunque sí práctico, al eslabonamiento de ambas actividades (es decir, a una progresión infinita). La cuarta actividad, a la que llamamos 'técni$\mathrm{ca}^{\prime}$, consiste en la ejecución de los procedimientos que ha diseñado quien se dedica a la tercera, lo que comúnmente conlleva el uso de artefactos. Quien se dedica a la primera actividad es científico; quien a la tercera, tecnólogo. Quien se dedica a la segunda ejerce a la vez de científico y de tecnólogo. La persona que realiza la primera versión de la cuarta, la fabricación de artefactos, es ingeniero; la que realiza la segunda versión, la ejecución de procedimientos mediante el uso de artefactos, es menestral. En ocasiones es el mismo ingeniero 
quien diseña y fabrica una herramienta, por lo que la frontera entre ambos roles se difumina. Además, todo ingeniero es a la vez menestral, a menos que fabrique las herramientas con la sola ayuda de sus manos.

Bunge previene contra el neologísmo 'tecnociencia', que confunde ciencia con técnica (2006b, 38; 2012a, 79). Cita el comentario de Jorge Sabato de que «la ciencia no segrega técnica» (2003b, 46). Así, en algunas ciencias, como la física nuclear, la mayoría de los artículos no prevén aplicaciones prácticas (2012a, 82). Por su parte, a la técnica, sea directa o indirectamente, todo conocimiento epistémico le es de utilidad; por eso no le hace falta pedirle a la ciencia que la provea de conocimientos aplicables: a esta le es imposible no hacerlo (1960a, 40).

\section{Dos funciones distintas}

La ciencia y la técnica se parecen en que son necesarias para la subsistencia y en que comparten el método científico. Las distingue su finalidad: la técnica, y con ella la tecnología y la ciencia aplicada, tiene una meta práctica; la ciencia investiga solo porque quiere saber (1980a, 212). Otra diferencia es metodológica: además de la eficacia, el tecnólogo procura también la eficiencia y por eso en ocasiones preferirá la simplicidad, la parcialidad y la superficialidad. Eso sí, de rechazar sus contrarios necesitándolos, sería un seudotecnólogo (1980a, 214). Al tecnólogo le interesan los resultados; apenas cómo los ha logrado. Si una caja negra funciona, su mecanismo le es indiferente (1972, 275). Una tercera diferencia es axiológica: los resultados científicos son todos neutrales, los técnicos poseen todos valor (idem, 276). Las técnicas son utilitarias, es decir, asignan valores a sus objetos de estudio; las ciencias son desinteresadas, es decir, no asignan a los suyos ningún tipo de valor (2012a, 81). El motor de la ciencia es la curiosidad; el de la técnica es la necesidad: «mientras que la predicción científica dice qué pasará o qué pasaría si se dan ciertas circunstancias, la tecnológica sugiere cómo influir en las circunstancias que modo que se puedan producir o prevenir ciertos efectos que normalmente no ocurrirían» (1967b, 156). Siendo tan diferentes, ciencia y técnica son complementarias: se deben combinar para evitar la especulación etérea, que acaba por versar sobre entelequias, y la acción sin guía, que termina en el estropicio (1966, 332).

Bunge juzga analética ${ }^{2}$ la técnica: las tesis descriptivas pueden ser más o menos verdaderas; las normativas, en cambio, más o menos eficaces. Propugna que las teorías técnicas son comprobables empíricamente, «pero no tan firmemente como las teorías científicas» (1967b, 137). La superación del estadio preparadigmático de la técnica consistió en pasar de las 'rules of thumb' a las

2. Alético: término adjetival correspondiente al término nominal 'verdad'. Analético: lo es aquello ajeno a la verdad y la falsedad. 
reglas fundamentadas en leyes científicas (1967b, 145), lo que supuso plantear las hipótesis sobre la eficacia como problemas de ciencia: nació la ciencia aplicada. Su fundamento es la conversión del problema inverso \{eficacia $₫$ verdad\} en el problema directo \{verdad $\otimes$ eficacia\} $(1967 b, 153)$. Tras exponer esas tesis, Bunge insiste en las profundas diferencias entre 'law formulas' y 'rules' (no queda claro si las reglas se enuncian o no mediante fórmulas) y deplora que su confusión sea persistente (ibidem). Rebatimos a Bunge: juzgamos que las hipótesis y tesis prácticas son de la misma clase que las teóricas y que considerarlas como clases distintas provoca una epistemología técnica errada.

Acertada o desacertada, la diferencia es clara: una regla «es una indicación» de cómo "proceder para conseguir un efecto determinado»; "una ley, en cambio, enuncia lo que algo es» (2012b, 62). Las tesis epistemológicas prescriptivas no pueden ser verdaderas ni falsas, «sino fructíferas, yermas, nocivas o entretenidas», y por tanto su comprobación consiste en su fertilidad, su vaguedad y su consistencia filosófica (1967a, 416); dicho de modo más breve, solo cabe juzgar si resultan 'fruitful' o 'fruitless' (idem, 306). Es uno de los asuntos en los que más insiste Bunge: «el valor supremo de la investigación científica básica es la verdad, mientras que el de la técnica es la utilidad. Demostración: las pruebas científicas son tests de verdad, mientras que las técnicas son tests de eficiencia» $(2002,45)$. La receta médica, la regla tecnológica o la norma moral no son proposiciones, sino propuestas. No son verdaderas o falsas, sino efectivas, inefectivas o contraproducentes (1983b, 140). Destacamos que Bunge sigue manteniendo esta tesis en la actualidad: de las teorías científicas se comprueba su verdad; de las teorías técnicas se comprueba su eficacia $(2017,152)$.

La tesis bungeana resulta alarmantemente parecida a la tesis central del pragmatismo; juzgamos que se trata del mismo error: confundir una propiedad ${ }^{3}$, el grado de éxito o fracaso, con la cualidad que es su causa. Propugna que «la búsqueda de la verdad no es lo mismo que la búsqueda de la utilidad» (1983b, 114). Rebatimos a Bunge: toda tecnología es búsqueda de conocimiento de tipo práctico, que necesariamente implica conocimiento de tipo teórico; la causa de la confusión es que no sucede igual a la inversa, porque parte del conocimiento teórico se busca para satisfacer la pulsión humana (quizá también de otras especies) de la pura curiosidad.

Creemos que Bunge se excede al distinguir la verdad de las teorías de la eficacia de los diseños. Proponemos la siguiente explicación. Un dispositivo es efectivo si mediante él se consigue aquello para lo cual se lo ha diseñado; es tanto más efectivo cuanto más o mejor logre desempeñar su función. Es tanto más eficaz cuantos más intentos de hacerlo funcionar tengan éxito o cuanto mayor sea el éxito de cada intento. Es tanto más eficiente cuanto menor sea

3. Proponemos el término 'propiedad' para el concepto tradicionalmente denominado 'disposición'. 
el insumo necesario para realizar un intento o para que el intento tenga éxito. El diseño de un dispositivo es una teoría conformada por tesis prácticas, cuya comprobación empírica es la ejecución del propio diseño más, en el caso de los artefactos, su utilización. Así, la experimentación mediante el uso de un dispositivo permite comprobar las tesis prácticas de la teoría que explica su diseño, las cuales se refieren a su efectividad, su eficacia y su eficiencia.

Bunge rechaza de plano nuestra explicación en (1983b, 60): «la verdad guía la busca de la eficiencia, y la eficacia plantea el problema de su explicación, pero se trata de categorías distintas. Tan es así, que la verdad es a menudo inútil, y la eficacia se basa a veces en la mentira». En cambio, en varios lugares el propio Bunge parece darnos la razón: «aunque las normas no se pueden someter a pruebas de verdad, sí se puede comprobar su congruencia (con otras normas en un sistema normativo determinado) y su eficiencia práctica» $(1996,318)$; «no hay un hiato insuperable entre leyes y reglas por cuanto, lejos de ser independientes de la voluntad humana, algunas reglas se vuelven leyes» (1985a, 159); el racioempirismo «exige que toda proposición, ya sea hipótesis o dato, así como toda norma se justifique teórica o empíricamente»(1996, 446). Sucede hasta tres veces en una página del Treatise: «la eficacia que se le supone a una regla debe comprobarse y no darse por segura»; «tampoco el éxito garantiza la verdad: tan solo plantea el problema de explicar por qué la propuesta en cuestión funciona»; "la relación entre conocimiento teórico y conocimiento práctico, entre ciencia y tecnología, y entre saber y hacer, están implicadas» (1983b, 143). Estas dos citas no propugnan sino nuestra tesis: «los componentes elementales de las cosas artificiales "obedecen" las leyes naturales -en tanto en cuanto cada regularidad artificial se puede analizar en leyes naturales-. (...) los tecnólogos pueden contribuir a la emergencia de nuevas leyes -aquellas inherentes al artefacto que diseñan- (...). Toda cosa artificial es un sistema con propiedades emergentes, y posiblemente también leyes emergentes; y todo proceso artificial es un cambio en un sistema tal. Sin embargo, los componentes elementales de una cosa artificial son cosas naturales que satisfacen leyes naturales; de igual modo que los componentes elementales de un proceso artificial son cosas naturales» (1985a, 225); "los sistemas técnicos, o dispositivos, difieren de los naturales en que son materializaciones de ideas (diseños). Pero, desde luego, se ajustan a leyes naturales» (2008a, 17). Además de lo anterior, creemos que el modelo epistemológico de Bunge es coherente con nuestra corrección, a pesar de que él esté convencido de lo contrario.

$\mathrm{Al}$ argumento bungeano de que las tesis de la técnica son hortatorias también se opone Seni (1990). Una teoría técnica explica la interacción agenteobjeto, mejor dicho, "la acción de un agente que afecta a un objeto», de modo que ambos constituyen «supersistemas agente-objeto». La clase de referencia de las teorías técnicas son «supersistemas cosa-artificial-y-agente». El autor expone una importante gestión conceptual: a 'ciencia' le corresponde 'teoría', y a 'tecnología' le corresponde 'plan'. Un 'plan' es «un constructo que describe el 
estado futuro de un objeto junto con la trayectoria que resulta de la acción [de alguien]».

Resulta cuando menos perturbador que en algún que otro lugar también lo diga Bunge: el dispositivo y el manipulador constituyen un suprasistema porque varios vectores del espacio de estados del objeto son acciones del hombre o sus 'proxies' (1985a, cap. 5). Por tanto, «el objeto y el sujeto -ahora un agente- no son separables: forman un supersistema» (1985a, 229). "Un plan (o programa) de un sistema en un periodo dado es una función de estado que prescribe la historia del sistema durante ese periodo como objeto de la acción (trabajo) de seres humanos o sustitutos suyos» (1985a, 230). Así, un plan, o programa, se compone de tareas, cada una de las cuales se compone a su vez de acciones que deben ser realizadas.

\section{Axiología y praxiología más otros valores}

La ciencia solamente pretende conocer; en cambio, la técnica tiene diversas pretensiones. La axiología consiste en evaluar lo pretensible; la praxiología consiste en evaluar los posibles medios de su consecución. Bunge llama a la axiología 'teoría de los valores' y la distingue de la 'ética' o 'filosofía moral' (2002, 32). A la praxiología la llama 'teoría de la acción' y la define como «la rama de la filosofía práctica interesada en la acción humana deliberada» (2006a, 377). Proponemos superar esta vaga definición con otra del propio Bunge, según la cual la praxiología investiga los «conceptos generales de la acción individual y la colectiva, así como las condición de la acción eficaz, sea cual sea su valor moral» (1999b). Acorde con ella, la axiología se encarga de valuar los fines, sin pretender en momento alguno valorarlos.

En esta parte del conocimiento aparece la ideología como cuestión: «del científico esperamos verdades y del tecnólogo esperamos diseños o planes que beneficien a alguien, de modo que lo observamos con la lupa moral» (2014, 364). Es habitual confundir la pareja axiología-praxiología con la ideología. Creemos que Bunge alterna tres soluciones a ese problema: (i) distingue, tácita o explícitamente, ambas partes; (ii) identifica la una con la otra; (iii) considera la segunda como un caso de la primera. Juzgamos acertada la primera solución y erróneas las otras dos.

Bunge intenta solucionar el trilema mediante una gestión conceptual. Llama 'endomoral' a la praxiología, compartida por la ciencia y la técnica, y 'exomoral' a las convicciones ideológicas, exclusivas de la técnica (1995, 119-121). Define 'exomoral' como «el conjunto de las normas morales que debieran regular el ejercicio de las profesiones técnicas para que éstas sirvan al público en lugar de servir exclusivamente intereses particulares» (ibidem). Su distinción (1989b, 200) entre 'exoaxiología' y 'endoaxiología' no hace sino demarcar lo axiológico y lo praxiológico. Creemos que las variaciones del par 'endo'-'exo' 
se deben a que intenta hacer corresponder tres elementos en una dicotomía. La solución (i), la que compartimos con él, resuelve el problema.

Bunge identifica axiología y praxiología con ideología:

el científico social no puede evitar hacer juicios de valor, como no puede evitar el ingeniero evaluar la eficacia de sus artefactos, o el médico la salud de sus pacientes. La única condición que esas evaluaciones deben satisfacer es la de ser objetivas. La noción de juicio de valor objetivo parece una contradicción en los términos solo porque domina una teoría de los valores idealista. Los biólogos lo saben mejor: saben que, mientras ciertas propiedades y procesos son buenos para el organismo, otros son malos. De igual manera, es fácil para un científico social mostrar que la desnutrición, la ignorancia y la opresión son malas para la gente que las sufre: no hay nada de partisano en ese juicio de valor. Por ello mismo, el científico social -a menos que esté al servicio de una dictadura- debe mostrarse de acuerdo en que una sociedad donde la gran mayoría está deprimida económicamente, privada de cultura u oprimida políticamente es objetivamente peor que una sociedad más equitativa. (1985a, 120).

Sin embargo, más adelante es del todo ideólogo; por ejemplo, en (1985a, 290): "la sociotecnología sin democracia no puede evitar la tiranía; y la democracia sin sociotecnología es ciega, y entonces ineficaz, y por tanto frágil»; (idem, 306): «maximizar la utilidad esperable es irracional en algunos casos e inmoral toda vez que implica dañar a otros»; e (idem, 308): «asegurar que se proteja el interés público». Incluso se resiste a separarlas en (1985a, 299): «la evaluación no es moralmente fiable, porque la encarga una de las partes (...) con razones para ser parcial, y porque los intereses creados y las ideologías tienden a desempeñar algún papel». En algunos lugares introduce la ideología en el sistema del conocimiento: «además, la epistemología, al estudiar las normas morales con las que se rige tanto el científico como el tecnólogo, adquiere un alto contenido ético» (1987b, 161); la praxiología «tiene en cuenta el valor moral de la acción humana. Es decir, distingue las buenas acciones de las malas» (2003a, 182). En otros resulta ambiguo: «la acción -en particular si está bien planeada- puede llevar de lo que es a lo que debería ser» $(2001,249)$.

En otras ocasiones considera que la ideología es un componente de la axiología, de la praxiología o de las dos. Según Bunge, «la praxiología, a diferencia de la sociología, tiene en cuenta el valor moral de la acción: distingue las buenas acciones de las malas y de las moralmente indiferentes» (2002, 51). Sucede así porque «un valor puede ser objetivo, subjetivo, o ambas cosas a la vez. (...) un objeto es objetivamente valioso si satisface algna necesidad o aspiración legítima (...) subjetivamente valioso si se lo desea independientemente de su valor objetivo» (2002, 43); "de ahí la necesidad de una investigación sin valores junto con una actuación política que sí los tenga (...). Eso sugiere una teoría de los valores 
que combine lo cognitivo con lo emotivo» (1999a, 307). La praxiología puede entenderse de manera "estrecha" si se considera como su objeto de estudio "determinar las condiciones para la acción eficiente, sin importar las consideraciones morales». Dejando aparte su importancia ideológica, no considerar la ética y la moral provoca, objetivamente, perder eficacia. Por tanto, propone juzgar buena la «acción que satisface dos condiciones: la condición técnica de eficiencia óptima y la condición moral de ser más beneficiosa que perjudicial» (2006a, 377-379).

En otros lugares las intenta articular. Las tesis pueden ser 'substantive' (vale decir teóricas) o 'nonsustantive', las cuales son «valuativas o perspectivas: guían la investigación y la acción». Las 'metodológicas' son las que rigen el procedimiento; una tesis 'axiológica', o 'valorativa', es «un juicio de valor», que consiste en dictaminar «que una teoría dada es a todas luces mejor que otra, o que cierta acción es buena, o un estilo de vida dado lo es». Lo que esperamos de las tesis metodológicas son criterios praxiológicos; lo que esperamos de las axiológicas es "que nos ayudan a elegir o hacer lo mejor o evitar lo peor» (1983a, 314). En el caso de las ciencias sociales, a la explicación (que se obtiene a partir de las leyes científicas junto con las circunstancias) se le pueden o no añadir «juicios de valor o normas de algún tipo» $(1997,442)$. De ese modo: «tesis o hipótesis \& juicio(s) de valor \& norma(s) \& circunstancias $\therefore$ explanando».

Quintanilla vacila entre esta postura y la nuestra $(2000,205)$ : «la praxiología no evalúa las acciones solamente desde el punto de vista de su eficiencia y consistencia. También propone el análisis de las condiciones que las limitan. Esto es particularmente importante dado que entre los condicionamientos a los que están sometidas las acciones hallamos los estándares éticos». Al considerar la discusión anterior igual que Bunge, Quintanilla propugna tres premisas de la acción: la epistemológica, la técnica y la ética; «se trataría de diseñar y desarroIlar propuestas tecnológicas no solo funcionales (eficientes y eficaces), [sino,] además, moralmente aceptables» (idem, 212).

Por último, y con acierto, en bastantes lugares considera la una y las otras como distintas e independientes. Esta postura suya es también la nuestra: toda tesis técnica tiene dos premisas: una axiopraxiológica y otra ideológica. La axiología y la praxiología son objetivas: «maximícese la exactitud de las predicciones y la definición de los medios y tendremos una toma de decisiones máximamente racional» $(1972,266)$. La praxiología no depende de la ideología: estudia los métodos plausibles de llevar a buen puerto cualquier empresa, sea esta honrosa o deleznable (1980a, 223). Si «los resultados de la investigación pura son éticamente neutros, como lo demuestra el hecho de que pueden ser utilizados para bien o para mal» (1960b, 56) y «una acción puede ser eficaz y satisfactoria para su agente, aunque moralmente errónea por ser egoísta, así como puede estar bien motivada moralmente pero ser ineficaz o contraproducente. Ello muestra que la ética y la praxiología no deben conducirse aisladas la una de la otra, como suele suceder. Solo la unión de los dos campos puede acometer los problemas de la legitimación completa -tanto práctica como moral- de la acción» (1999b), enton- 
ces «todo asunto social tiene un aspecto moral, es decir, involucra un problema moral añadido al práctico, ya que trata de derechos y deberes, enraizados en necesidades y deseos» (1989a, 354).

De todo lo anterior se desprende que las normas, consistan en un hágase o en un impídase, tienen dos clases de premisas: las praxiológicas y las ideológicas $(1989 a, 331 ; 2004,181)$. Bunge lleva estas consideraciones a la tesis de que la ideología es mala, o al menos peligrosa. En el Treatise distingue conocimiento de ideología, desacreditanto esta: «los problemas políticos pueden tratarse ideológica o científicamente. El primer procedimiento consiste en seguir una estrategia sugerida por una intuición, por creencias que no se examinan o por intereses creados. El método científico consiste en diseñar un programa social encaminado a resolver el problema, a la luz de un estudio científico cuidadoso de la situación» (1983b, 230). Así, la ideología es un agente externo a la ciencia que se instila en ella y la perjudica: (i) la ideología personal influye en lo que toda persona hace, incluida la ciencia; (ii) ningún elemento ideológico es un constituyente de la actividad científica; por tanto, (iii) se trata de sesgos que hay que eliminar o, de no ser eso posible, paliar; (iv) si un elemento ideológico influye de manera significativa en un acto científico, entonces el resultado de ese acto científico pierde calidad, en ocasiones hasta el punto de resultar inaceptable (1991, cap. 1).

De esto se sigue la recomendación bungeana de «eludir la ideología en la ciencia básica pero buscarla en todo lugar, y declarar con franqueza tus valores sociales en ciencia aplicada y tecnología, especialmente en tecnología social» (1983b, 270; 1985c, 51). Con la segunda parte de nuestro estudio pretendemos demostrar que esta idea es desacertada por completo, y que ese fallo impide el progreso de la filosofía y de todas las demás disciplinas, que dependen de ella.

Creemos ver en la doctrina de Bunge una tesis medular de la nuestra: parte de lo que se considera ideología es realmente ciencia ética, vale decir axiología y praxiología. «En ninguna situación podemos atenernos a la máxima de que en la ciencia social deben estar ausentes los valores: si nos empeñamos en ello, los humanos nos parecerán autómatas (...). La solución no está en ignorar los valores, sino en estudiarlos científicamente y entonces avanzar hacia una fusión de la sociología y la teoría de los valores» (1985a, 161). Propugnamos que muchas cuestiones que se consideran como ideológicas pueden analizarse científicamente, con lo cual dejarían de ser convicciones y pasarían a ser nociones ${ }^{4}$. Eso coincide con (2006b, 130), «las normas morales no son a priori, puesto que se refieren a la vida real, y por lo tanto debieran ser puestas a prueba del mismo modo que las hipótesis científicas», y con $(2002,33)$, «es posible y deseable justificar racional y empíricamente la elección de valores, así como elaborar

4. Distinguimos 'nociones' y 'convicciones' en la tercera sección. 
reglas de conducta que incorporen valores que han sido pasados por los tamices de la ciencia y la ténica».

Las ciencias humanas son necesarias para que las normas técnicas sean justas y eficaces (2010a, 53). Corregimos: para que sean eficaces, sí; para que sean justas hace falta algo más. Eso que falta lo expone en la misma página: los valores tienen sus raíces en los entes psíquicos y sociales; las normas son mejores si benefician al cuerpo y la mente de la persona en cuestión y los cuerpos y mentes de las otras personas con las que ella convive. Comulgamos con esa tesis y con otra que se desprende de ella: la axiología y la praxiología son necesarias y suficientes para regir las acciones; la ideología es sobrante, pero no prescindible.

Bunge llega a una consecuencia que compartimos con él: «un programa social o un plan podría llamarse 'científico' si, y solo si, se basa en (a) teorías científicas, modelos y datos, así como en (b) un sistema de valores compatible con el conocimiento científico actual» (1985a, 290); con lo que «es posible y deseable intentar fundamentar la axiología y la ética sobre la ciencia y la técnica, en lugar de sostener que los valores y las reglas morales son puramente emotivos, o convenciones sociales (...). Por ejemplo, se puede argüir en favor de la retribución justa del trabajo, recurriendo no sólo a los sentimientos de compasión y solidaridad, sino también a las estadísticas que muestran que la longevidad y la productividad aumentan con el ingreso. O sea, la justicia social es un buen negocio» (2006b, 129). "Las "razones" de marras deben ser sólidos hallazgos de las ciencias sociales, reglas sociotecnológicas eficaces, datos empíricos firmes y principios morales universales» $(2001,265)$.

De las muchas tesis consecuentes con lo anterior que salpimentan la obra bungeana, ofrecemos dos como botón de muestra: «verter agua tóxica en un lago es un hecho moral (...) porque viola el derecho de otros a disfrutar de un entorno limpio (...) por consiguiente, la proposición "verter agua tóxica en un lago está mal" es una verdad moral» (1989a, 308); "una ideología sociopolítica científica preconizará una democracia integral o participativa en lugar de una democracia meramente representativa o, aún peor, de una dictadura» (1980b, 100). En (1990, 648) es explícito: todo plan presupone un sistema de valores y un sistema de principios morales que lo subyace. Ya que los valores y los principios morales influyen en las premisas técnicas, deben examinarse tan cuidadosamente como se examinan estas.

Aunque a veces parezca compartir nuestra postura, la prédica de Bunge es ambivalente: «la ciencia y la técnica ayudan a resolver problemas morales, al proveer medios, pero no suplen las reglas morales. Estas emergen y se modifican en la vida social y en la reflexión acerca de ella» (2014, 351); sin embargo, en el mismo parágrafo termina diciendo: «puesto que la ética estudia las normas que guían las acciones humanas, que procuran realizar valores, como el bienestar y la convivencia, la ética debería fundarse sobre la axiología, o teoría de los valores, como también sobre la praxiología, o teoría de la acción». 


\section{La ideología}

El ingrediente de las teorías técnicas que la ciencia no puede aportar es ideológico. Postulamos que la disciplina que lo estudie no puede ser científica, aunque tampoco debe se inconsistente con la ciencia. Para explicar qué es y cómo es la ideología nos tenemos que retrotraer a la racionalidad, que a continuación explicamos.

\section{La racionalidad}

La facultad de razonar se denomina 'raciocinio', igual que la de hablar se Ilama 'lenguaje'. Un 'razonamiento' es un acto de razonar, y consiste en realizar una colección de inferencias; cuando se habla del 'razonamiento' en general, el referente son los actos de ese tipo que realizan la persona o las personas en cuestión. La 'racionalidad' es una cualidad que necesariamente posee toda inferencia, en mayor o menor grado. El intervalo ínfimo de racionalidad se denomina 'irracionalidad'. Se dice de una persona, por metonimia, que es más o menos racional según la proporción de inferencias suyas que son racionales y la proporción de las que son irracionales (cfr. Mosterín, 1978, 60). De igual modo que las lenguas no son innatas, sino que lo es el lenguaje, el razonamiento que cada individuo ejerce no es innato, sino que es el desarrollo -adquisición- a partir del estado congénito de una cualidad, ahora sí, innata: el raciocinio (cfr. Malabou, 2014).

La inteligencia es la capacidad de inteligir, que etimológicamente significa 'inter-ligar', es decir, anudar inferencias entre sí. Así, "consideramos que una persona es inteligente cuando razona con rapidez, seguridad y acierto» (Morgado, 2007, 157). Dicho de otro modo, la inteligencia es la capacidad de operar con los componentes de la razón. Sin embargo, se puede ser inteligente e irracional (normalmente, la irracionalidad pasa inadvertida para uno mismo). Stanovich (en Manktelow, 2012, 259) apunta «que ningún test de inteligencia mide el tipo de habilidades que se necesitan para pensar racionalmente». Denominamos 'logos' a la facultad de pensar, Ilamada 'pensamiento', y a la facultad de comunicar lo que se piensa mediante actos de comunicación complejos, llamada 'lenguaje', consideradas conjuntamente. Opugnamos la postura cognicista (mal Ilamada 'ciencista'), muy arraigada en la cultura occidental, que identifica el logos con la cognición. Postulamos, en cambio, un raciocinio diverso que incluye el conocimiento como una de sus partes.

Los componentes de la razón son las nociones, los sentimientos, las convicciones, las voliciones y las intenciones. Consideramos el psicón como unidad de pensamiento (1980c; 1987a); por mor de la brevedad, en este estudio no atendemos a la complejidad de los psicones. Una 'noción' es un psicón con el que un animal, merced a la percepción, aprehende un elemento de la realidad (eventualmente, un elemento imaginario). Una 'emoción' es la percepción de 
un estado somático; un 'sentimiento' es un psicón que contiene al menos una noción y al menos la aprehensión de una emoción (Damásio, 1994). Un 'apetito' es una emoción primitiva consistente en una proacción ${ }^{5}$ somática activada por una carencia (Damásio, 2003, 34); un 'deseo' es la asociación de un apetito a un objeto. Una 'volición' es un psicón que contiene al menos una noción y al menos un deseo. Un 'interés' es una volición cuyo deseo supone una necesidad para el animal. Una 'intención' es una volición más la imaginación de una acción. Una 'acción' es un movimiento o un cambio somático que es consecuencia de una intención y una decisión. Una 'conación' es una acción que consiste en producir una acción de otro animal, la cual satisfaga un deseo; por extensión, Ilamamos así a las intenciones que originan tales acciones. Una 'Convicción' es un psicón que contiene al menos una noción, al menos un sentimiento y al menos una volición.

La colección de todas las nociones de un animal se denomina 'nocionario'; la de todas las emociones y los sentimientos, 'emocionario'; la de todas las convicciones, 'conviccionario'. Al conjunto de todas las voliciones de un animal lo llamamos 'volicionario'. Descartamos 'acervo comportamental', término etológico que denota el conjunto de todas las acciones, actuales o potenciales, de un individuo, una población o una especie.

Una 'inferencia' consiste en elaborar una o varias nociones, emociones, convicciones, voliciones o acciones ${ }^{6}$ a partir de varias de las cuatro primeras. El razonamiento consiste no solo en la inferencia de "unidades semánticas", sino también de "unidades pragmáticas" (Habermas, 1981, 43). Por eso, los promotores de la lógica informal propugnaban: (i) que la lógica deductiva y la lógica inductiva estándar son insuficientes para la argumentación, y (ii) que hay otros mecanismos inferenciales, distintos de los de ellas, que decididamente son formales y que permiten validar e invalidar argumentos. En una de sus obras fundacionales, Bunge propugna igual: «valores situados en niveles diferentes: el estético, perteneciente a la esfera de la sensibilidad, y el gnoseológico, que, no obstante estar enraizado en la sensibilidad, está enriquecido con una cualidad emergente: la razón»(1959a, 58; 1960a, 43), pero su postura se va endureciendo con el tiempo: «un razonamiento válido es una deducción de un conjunto de proposiciones de acuerdo con las reglas de la lógica deductiva. A veces, los razonamientos no deductivos, tales como los análogos y los inductivos, son fructíferos; pero carecen de validez formal»(1987a, 58).

5. Un mecanismo es 'proactivo' si sus productos (o 'output') causan que aumenten sus estímulos (o 'input'), los cuales a su vez hacen que aumenten los productos. Es 'retroactivo' si sus productos causan que disminuyan sus estímulos, los cuales a su vez hacen que disminuyan los productos.

6. Cuando decimos -forzadamente- "elaborar una acción", queremos decir elaborar los psicones que causen las eferencias que produzcan esa acción. 
De igual modo que hay metanociones, que constituyen el metaconocimiento o epistemología, hay metasentimientos (Castilla, 2000) y hay metaconvicciones (Wilson et al., 1998). Por su parte, los metafines son «fines generales que de algún modo regulan, ordenan, estructuran y restringen los fines concretos» (Mosterín, 1978, 82).

\section{Distinguir conocimiento de ideología}

Tenemos que resolver una confusión grave: la del concepto 'ideología' con el significado de 'ideario esclerosado'. En realidad, no se trata de una dilogía, sino de un caso de la categoría 'ideología' al que desgraciadamente se le asigna la misma palabra. Así, Bunge dice que «las ideologías son respuestas prefabricadas a estímulos esperables» $(2010 b, 199)$ y que el fundamentalismo consiste en el esclerosamiento consciente y deseado (cfr. 1985b, 150). Una persona es dogmática si, al inferir, considera algunas nociones con un grado de certidumbre mayor que el que tienen para ella o algunas convicciones con un grado de intensidad mayor que el que tienen para ella (cfr. Mosterín, 1978, cap. 1). Es escéptica si las considera con un grado de certidumbre o de intensidad menor. Una persona es más o menos crítica, según sea mayor o menor su capacidad de alterar su nocionario o su conviccionario como consecuencia de las experiencias cognitivas o ideológicas que experimente. Es frívola si acepta nociones o convicciones al tuntún, despreocupándose de cómo sea el nocionario o el conviccionario que resulte de ello (ibidem).

Quizás el error radical sea propugnar que una ideología es «un sistema de enunciados fácticos y juicios de valor» (1999a, 136). Objetamos y ampliamos: es un sistema de juicios de valor sobre enunciados fácticos; en tal sistema participan tres clases de componentes: nociones, sentimientos e intereses. A partir de estos componentes, ordenados de aquel modo, se generan las convicciones. Así, son requisitos de las convicciones ${ }^{7}$ (Nussbaum, 2001, 4): (i) «una apreciación cognitiva» de los elementos del universo; (ii) «la idea del propio florecimiento y de los propios objetivos y proyectos importantes»; (iii) "la idea de la prominencia de ciertos objetos como elementos del propio esquema de objetivos». Todo lo anterior nos permite analizar las ideologías en tanto que elementos de la realidad. Confutamos la óctupla de Bunge (2008b, cap. 4) y proponemos: (1) sociedad; (2) comunidad de correligionarios (extendemos el campo de referencia de este término); (3) base filosófica; (4) cosmovisión; (5) conviccionario; (6) problemario; (7) objetivos; (8) medios. Este resumen mejora otros anteriores, con los que es consistente: (1980a, 165; 1980b, 95-96; 1985b, 147-150). "Hasta ahora todas las culturas han sido mezcolanzas de elementos racionales e irracionales, con abrumador predominio de los segundos. La no-

7. Que no, de los sentimientos, como propugna la autora. 
vedad, fuerza y eficacia de nuestra cultura estriba precisamente en el decisivo desarrollo de algunos de sus componentes racionales» (Mosterín, 1978, 68).

Proponemos dos gestiones conceptuales y terminológicas distintas de las tradicionales. La 'cosmovisión' de una persona o una comunidad es su nocionario más las experiencias que ha vivido ${ }^{8}$. El 'lebenswelt' de una persona o una comunidad es su conviccionario más las experiencias que ha vivido ${ }^{9}$. Eso supera el concepto tradicional de 'ideario': \{nocionario + conviccionario + emocionario + volicionario\}.

Mosterín llama 'plan de vida' al conjunto de todos los planes de una persona en un instante o lapso determinado (Mosterín, 1978, 85). Los planes de vida son más o menos consistentes con la cosmovisión y el lebenswelt. Los metafines cohesionan los diversos planes y los hacen más coherentes, aunque en ocasiones los esclerotizan. Empecinarse en uno o varios fines es ser tozudo; obcecarse con uno o varios metafines lo convierte a uno en mártir (idem, 89). Un 'relato' es una colección, más o menos consistente, de fragmentos de una o varias cosmovisiones y uno o varios lebenswelten que se elabora con el propósito de fomentar o socavar una ideología determinada ${ }^{10}$.

\section{Breve explicación de la ideología}

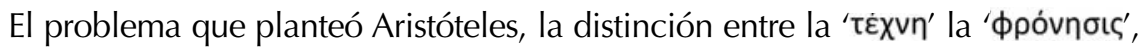
no ha sido resuelto (Gadamer, 1960, 512). A partir de lo anterior, proponemos la siguiente solución: la 'lógica' es la parte del raciocinio que se ocupa de las inferencias en las que participan mayoritariamente nociones; la 'frónesis', de las que se componen en su mayor parte de convicciones. Según Piaget (en Habermas, 1999, 270), el niño va desarrollando mecanismos de enjuiciamento moral para desenvolverse en la sociedad, de igual modo que desarrolla mecanismos de enjuiciamiento cognitivo para desenvolverse en la realidad. La cognición y la emoción tienen cada una su propia "gramática": «para la gramática cognitiva las cosas son como percibimos que son»; «para la gramática afectiva las cosas son como deseamos que sean» (Castilla, 2000, 188).

Las emociones y los sentimientos son participantes indispensables de la toma de decisiones (Damásio, 2003, 145). En toda inferencia afectiva participa la cognición y en toda inferencia cognitiva participa la emoción; hay, además,

8. En el caso de la comunidad, se entiende: el conjunto de las que han vivido todos sus miembros. Igual para el lebenswelt.

9. Habermas le da un significado más amplio y algo vago: «acervo culturalmente transmitido y lingüísticamente organizado de patrones de interpretación» (en McCarty, 1978, 465).

10. Aceptamos 'narración' y 'discurso' como sinónimos. Las tres acepciones son traslaticias y su distribución es muy similar; elegimos 'relato' por gusto personal y por ser, quizás, la palabra que menos dilogías pueda causar. 
muchos procesos mixtos (cfr. Pessoa, 2008). La distribución anatómica de ambas clases de inferencias no es estanca, y topológicamente hay pocas diferencias. En esos procesos mixtos no están acopladas, sino integradas. Muchos estudios prueban la sinergia emocognitiva (Cornelius, 1996, 229). El ser humano dispone de un «sistema natural de navegación emocional» (Damásio, 2003, 157). Si ese sistema se estropea, la persona no categoriza correctamente, o sabe hacerlo, pero no toma decisiones correctamente. "Las emociones facilitan el razonamiento y ayudan a decidir y planificar el futuro» (Morgado, 2007, 102). Al ser más eficiente («más fluido») el razonamiento, la persona es más eficaz tomando decisiones.

Kahneman propone una distinción en $(2011,534)$ : una persona es 'razonable' «si sus creencias concuerdan por lo general con la realidad y si sus preferencias están en consonancia con sus intereses y valores»; es 'racional' "si sus creencias y preferencias son internamente consistentes», es decir, independientemente de si son consistentes con la realidad y con el emocionario y el volicionario que, respectivamente, las generan. Añade después que 'irracional' es el contrario de 'razonable', no de 'racional'. Así, una persona razonable «no puede ser racional por definición»; por tanto, el modelo de agente racional es erróneo. Su racionalidad se corresponde con nuestras axiología y praxiología (si se prefiere, con el concepto 'zweckrationalität'). Su razonabilidad es el fundamento ideológico de la acción (o 'wertrationalität'). En contra de Kahneman, postulamos que ambas clases de razonamientos son racionales y que la mala ejecución de cualquiera de ellas es irracional.

Es notorio que Bunge coincida con nuestras tesis en algunos lugares: «¿Qué hay de la teoría de los valores, la estética y la ideología?, ¿se las puede considerar tipos de conocimiento?; y, de ser así, ¿qué lugar ocupan en nuestro esquema? De primeras, ninguna lo es, porque todas se ocupan de valores, los cuales no son actos cognitivos aunque se realicen a la luz del conocimiento. Sin embargo, no todo enunciado de la teoría de los valores, la estética, la ética o la idología es un juicio de valor: también hay enunciados estrictamente descriptivos o explicativos en esos campos» (1983b, 196); "como nieto de un magistrado liberal y como hijo de un parlamentario progresista, no podría estar más de acuerdo» (2009, 181); «si izquierdista significa estar del lado de los oprimidos, pido que me declaren culpable» (idem, 189).

\section{Objetivo y subjetivo}

La ideología, como estudio de las convicciones, debe combinar la objetividad con la subjetividad. Empero, la labor subjetiva no debe confundirse con el estudio objetivo de lo subjetivo. Ricœur (en Ferraris, 1988, 389) propugna que un texto se puede indagar hermenéuticamente, en tanto que «huella de una palabra viva», o científicamente, en tanto que «objeto autónomo». 
Bunge, por el contrario, propugna que no atenerse a la objetividad implica irracionalidad $(1996,217)$ y afirma que «un realista u objetivista debiera admitir la pertinencia de los sentimientos, las opiniones y los intereses a la acción social, pero insistirá en que sean estudiados objetivamente» $(1995,176)$. Sin embargo, en ocasiones se alinea inopinadamente con nuestra tesis: «esta conclusión habría disgustado a Leibniz, que soñaba con remplazar toda disputa por un cálculo. Pero es un hecho que, en cuestiones de valores, el cálculo puede complementar la argumentación, la experiencia y el sentimiento, pero no remplazarlos» (1989b, 90).

\section{La honradez y la honestidad}

Una cuestión fundamental en el estudio y la gestión de la ideología es exponer las convicciones, personales o compartidas, que fundamentan los argumentos axiopraxiológicos en toda explicación técnica. Las explicaciones técnicas, que constan de dos fases, requieren honestidad para la primera y honradez para la segunda. Es habitual faltar a una o a otra, sobre todo en cuestiones económicas y políticas. Por ejemplo, si se proponen medidas que se sabe que provocarán la desdicha de muchas personas, se suele faltar a la honradez al ocultar ese hecho. Si ese hecho es inocultable, se suele decir que no hay opción mejor, aunque se desearía. Esto último es faltar a la honestidad: quien propone esa medida no expone la propia convicción de que las aflicciones del grueso de la población, o de los grupos sociales afectados, le son indiferentes, o le causan pena, pero una pena pequeña. No es cierto que no sepa que hace daño; hace daño y juzga que está bien hacerlo.

Habermas define la honestidad como «la manifestación de los propios deseos e inclinaciones, de los propios sentimientos y estados de ánimo», ya que «en muchas situaciones un actor puede tener muy buenas razones para ocultar sus vivencias a los otros o para despistarlos» (1981, 41; cfr. 1999, 118). En algunos lugares, llama 'verdad' a nuestra 'honradez' y 'veracidad' a nuestra 'honestidad' (vg., 1984, 358). Al igual que la frónesis, son una competencia práctica; se adquieren en contacto con gente prudente. Buen ejemplo de deshonestidad es la obra de Hayek (cfr. Bunge, 2008b; otros lugares). Suele acompañarla en sus correrías la falta de honradez.

\section{Conclusión}

Creemos que la ideología, tal como la explicamos y la sustentamos en su sección de este artículo, constituye la clase de razones axiológicas que Bunge echa en falta en su teoría técnica. Creemos que elucidar esta cuestión supone solucionar un problema importante para la sociedad actual, muy tecnificada y a la vez muy descuidada de sus valores. Saber exponer con honestidad la valoración de los argumentos y saber exponer con honradez la evaluación de las 
propuestas hará más eficaz la toma de decisiones, en esta época en la que las consecuencias de lo que se decide repercuten grandemente en la vida de todos.

\section{Bibliografía}

BUNGE, Mario (1959). Metascientific queries. Charles C. Thomas, Springfield.

BUNGE, Mario, 2013 (1960a). La ciencia - Su método y su filosofía. Laetoli, Pamplona.

BUNGE, Mario, 1996 (1960b). Ética, ciencia y técnica. Sudamericana, Buenos Aires.

BUNGE, Mario (1966). "Technology as applied science". Technology and Culture, 7 (3), 329-347.

BUNGE, Mario, 1998 (1967a). Philosophy of science, t. 1. Transaction publishers, New Brunswick.

BUNGE, Mario, 1998 (1967b). Philosophy of science, t. 2. Transaction publishers, New Brunswick.

BUNGE, Mario, 1975 (1972). Teoría y realidad. Ariel, Barcelona.

BUNGE, Mario (1976). Tecnología y filosofía. Universidad Autónoma de Nuevo León, México.

BUNGE, Mario (1980a). Epistemología - Curso de actualización. Ariel, Barcelona.

BUNGE, Mario, 2014 (1980b). Ciencia, técnica y desarrollo. Laetoli, Pamplona.

BUNGE, Mario, 2011 (1980c). El problema mente-cerebro. Tecnos, Madrid.

BUNGE, Mario (1983a). Treatise on basic philosophy. T. 5. Exploring the world. Reidel, Dordrecht.

BUNGE, Mario (1983b). Treatise on basic philosophy. T. 6. Understanding the world. Reidel, Dordrecht.

BUNGE, Mario (1985a). Treatise on basic philosophy. T. 7. Philosophy of science and technology. Reidel, Dordrecht.

BUNGE, Mario, 2013 (1985b). Pseudociencia e ideología. Laetoli, Pamplona.

BUNGE, Mario (1985c). Racionalidad y realismo. Alianza, Madrid.

BUNGE, Mario; Ardila, Rubén, 1988 (1987a). Filosofía de la psicología. Ariel, Barcelona.

BUNGE, Mario (1987b). Vistas y entrevistas. Siglo Veinte, Buenos Aires.

BUNGE, Mario (1989a). Treatise on basic philosophy. T. 8. Ethics: the good and the right. Reidel, Dordrecht.

BUNGE, Mario (1989b). Mente y sociedad. Alianza, Madrid.

BUNGE, Mario (1990). "Mario Bunge replies". En Paul Weingartner; Georg J. W. Dorn (Eds.), Studies on Mario Bunge's Treatise. Rodopi, Ámsterdam. 
BUNGE, Mario, 2015 (1991). Crítica de la nueva sociología de la ciencia. Laetoli, Pamplona.

BUNGE, Mario, 1999 (1995). Sistemas sociales y filosofía. Sudamericana, Buenos Aires.

BUNGE, Mario, 1999 (1996). Buscar la filosofía en las ciencias sociales. Siglo $\mathrm{XXI}$, México.

BUNGE, Mario (1997). "Mechanism and explanation". Philosophy of the Social Sciences, 27 (4), 410-465.

BUNGE, Mario, 2003 (1999a). Philosophical dictionary. Prometheus Books, Nueva York.

BUNGE, Mario, 2001 (1999b). "The technologies in Philosophy". En Martin Mahner (Comp.), Scientific realism - Selected essays of Mario Bunge. Prometheus, Amherst.

BUNGE, Mario (2000). "Ten models of individualism (none of which works) and their alternatives". Philosophy of the Social Sciences, 30, 384-406.

BUNGE, Mario, 2002 (2001). Crisis y reconstrucción de la filosofía. Gedisa, Barcelona.

BUNGE, Mario (2002). Ser, saber, hacer. Paidós, México.

BUNGE, Mario, 2007 (2003a). Una filosofia realista para el nuevo milenio. Universidad del Inca Garcilaso de la Vega, Perú.

BUNGE, Mario (2003b). Cápsulas. Gedisa, Barcelona.

BUNGE, Mario; Mahner, Martin (2004). Über die Natur der Dinge. Hirzel, Stuttgart.

BUNGE, Mario, 2007 (2006a). A la caza de la realidad. Gedisa, Barcelona.

BUNGE, Mario, 2014 (2006b). 100 ideas. Laetoli, Pamplona.

BUNGE, Mario (2008a). Filosofía y sociedad. Siglo XXI, México.

BUNGE, Mario, 2009 (2008b). Filosofía política. Gedisa, Barcelona.

BUNGE, Mario (2009). ¿Qué es filosofar científicamente? Universidad del Inca Garcilaso de la Vega, Perú.

BUNGE, Mario, 2015 (2010a). Materia y mente. Laetoli, Pamplona.

BUNGE, Mario (2010b). Las pseudociencias, jvaya timo! Laetoli, Pamplona.

BUNGE, Mario, 2015 (2012a). Evaluando filosofías. Gedisa, Barcelona.

BUNGE, Mario (2012b). Filosofía de la tecnología. Universidad del Inca Garcilaso de la Vega, Perú.

BUNGE, Mario (2014). Entre dos mundos. Memorias. Gedisa, Barcelona.

BUNGE, Mario (2017). Doing science in the light of philosophy. World Scientific, Singapur. 
CASTILLA DEL PINO, Carlos, 2008 (2000). Teoría de los sentimientos. Tusquets, Barcelona.

CORNELIUS, Randolf (1996). The science of emotion. Prentice-Hall, Upper Saddle River.

DAMÁSIO, António, 1996 (1994). Descartes' error - Emotion, reason and the human brain. Papermac, Londres.

DAMÁSIO, António (2003). Looking for Spinoza - Joy, sorrow, and the feeling brain. Harcourt, Orlando.

FERRARIS, Maurizio, 2000 (1988). Historia de la hermenéutica. Akal, Madrid.

GADAMER, Hans Georg, 1984 (1960). Verdad y método. Sígueme, Salamanca.

HABERMAS, Jürgen, 1999 (1981). Teoría de la acción comunicativa. T. 1. Taurus, Buenos Aires.

HABERMAS, Jürgen, 1989 (1984). Teoría de la acción comunicativa - Comentarios y estudios previos. Cátedra, Madrid.

HABERMAS, Jürgen, 2002 (1999). Verdad y justificación. Trotta, Madrid.

KAHNEMAN, Daniel, 2012 (2011). Pensar rápido, pensar despacio. Debate, Barcelona.

MANKTELOW, Ken (2012). Thinking and reasoning. Psychology Press, Hove.

MCCARTHY, Thomas, 1987 (1978). La teoría crítica de Jürgen Habermas. Tecnos, Madrid.

MORGADO, Ignacio, 2010 (2007). Emociones e inteligencia social. Ariel, Barcelona.

MOSTERÍN, Jesús, 1987 (1978). Racionalidad y acción humana. Alianza, Madrid.

NUSSBAUM, Martha (2001). Upheavals of thought - The intelligence of emotions. Cambridge University Press.

ORTEGA Y GASSET, José, 2015 (1939). Meditación de la técnica. Biblioteca Nueva, Madrid.

PESSOA, Luiz (2008). "On the relationship between emotion and cognition". Nature Reviews Neuroscience, 9, 148-158.

QUINTANILLA, Miguel Ángel (2000). "El concepto de eficiencia técnica". En Guillermo Denegri; Gladys Martínez (Eds.), Tópicos actuales de filosofía de la ciencia - Homenaje a Mario Bunge. Universidad Nacional de Mar del Plata.

SENI, Dan Alexander (1990). "The sociotechnology of sociotechnical systems - Elements of a theory of plans". En Paul Weingartner; Georg J. W. Dorn (Eds.), Studies on Mario Bunge's Treatise. Rodopi, Ámsterdam.

WILSON, Timothy; Gilbert, Daniel; Wheatley, Thalia (1998). Protecting our minds - The role of lay beliefs. En Vincent Yzerbyt et al. (Eds.), Metacognition. SAGE, London. 